\title{
Bluetooth Low Energy for autonomous Human-Robot Interaction
}

\author{
Marcus M. Scheunemann \\ marcus@mms.ai
}

\author{
Kerstin Dautenhahn \\ k.dautenhahn@herts.ac.uk
}

\author{
Adaptive Systems Research Group \\ University of Hertfordshire \\ AL10 9AB, Hatfield, UK
}

\begin{abstract}
This demonstration shows how inexpensive, off-the-shelf, and unobtrusive Bluetooth Low Energy (BLE) devices can be utilized for enabling robots to recognize touch gestures, to perceive proximity information, and to distinguish between interacting individuals autonomously. The received signal strength (RSS) between the BLE device attached to the robot and BLE devices attached to the interacting individuals is used to achieve this. Almost no software configuration is needed and the setup can be applied to most everyday environments and robot platforms.
\end{abstract}

\section{Keywords}

Bluetooth Low Energy; autonomous interaction; proxemics; human-robot interaction; robot design

\section{DESCRIPTION OF THE DEMO}

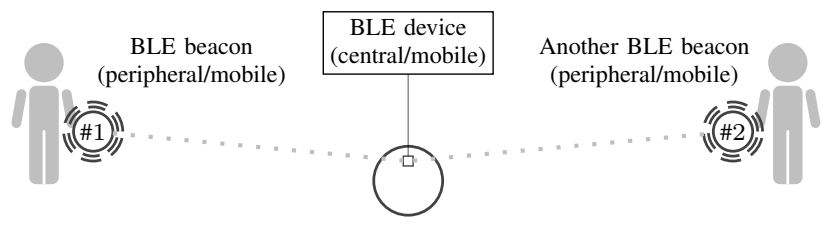

Figure 1: Example setup with two BLE beacons (i.e. peripheral devices) and one central device.

Figure 1 shows the overall concept. Interacting individuals wearing a pocket wristband equipped with a BLE beacon. Each beacon (i.e. peripheral device) advertises its ID every $100 \mathrm{~ms}$.

The mobile, rotating robot is equipped with a central BLE device. The central device is a system-on-chip (SoC) with a full BLE stack, making the computation completely independent of other hardware. The central BLE device scans passively and extracts the beacon ID and RSS data of the received advertisements of the beacons.

Permission to make digital or hard copies of part or all of this work for personal or classroom use is granted without fee provided that copies are not made or distributed for profit or commercial advantage and that copies bear this notice and the full citation on the first page. Copyrights for third-party components of this work must be honored. For all other uses, contact the owner/author(s).

HRI '17 Companion March 06-09, 2017, Vienna, Austria

(C) 2017 Copyright held by the owner/author(s).

ACM ISBN 978-1-4503-4885-0/17/03.

DOI: http://dx.doi.org/10.1145/3029798.3036663
The robot controller running on an external machine is connected via BLE to the central device and receives packages containing a beacon ID (1..n) and the corresponding RSS data to change the robot's behavior accordingly.

In the demonstration people are encouraged to interact with the robot. The mobile robot will emit differently colored light in accordance with the interacting person. We will provide pocket wristbands carrying BLE beacons. The RSS data between the central device on the robot and the beacons placed in the pocket wristbands can be observed on a screen. This provides insights of the reliability of the setup, the (non-linear) relation between distance of BLE devices and the RSS, as well as the impact of occluding body parts on the signal strength. A more in-depth explanation of the technology, the code for the central device, and a video of the overall experiment can be found in $[1,2]$.

\section{IMPORTANCE AND USEFULNESS}

All devices can be powered by a coin-cell battery. The computing of IDs and corresponding RSS data of surrounding beacons does not require an external machine. Thus, the beacons and the scanning device can be flexible mounted to robots or other objects. Therefore the technology allows quick prototyping of HRI experiments with autonomous robots. For example, in cases where the computer board of a robot cannot be amended with additional sensors this technology can still be applied.

The beacons need to be attached to the wrist or hand of the interacting individuals, but no further setup or configuration is needed. In addition, there are very few environmental constraints allowing for more autonomous HRI experiments outside a laboratory setting. Furthermore, the use of off-the-shelf components makes the overall setup affordable and easily applicable.

To the best of the authors' knowledge, we were the first using BLE for robots for deriving touch information from or for distinguishing between interacting individuals [2].

\section{REFERENCES}

[1] M. M. Scheunemann. BLE4HRI project page. Online, http://mms.ai/BLE4HRI, Dec 2016.

[2] M. M. Scheunemann, K. Dautenhahn, M. Salem, and B. Robins. Utilizing Bluetooth Low Energy to recognize proximity, touch and humans. In 2016 25th IEEE International Symposium in Robot and Human Interactive Communication (RO-MAN), Aug 2016. 